\section{EL DÍA MUNDIAL DE LA SALUD, LOS DETERMINANTES SOCIALES Y LAS OPORTUNIDADES}

\section{ABOUT THE WORLD HEALTH DAY, THE SOCIAL DETERMINANTS AND THE OPPORTUNITIES}

\author{
Diego A. Bernardini-Zambrini ${ }^{1, a}$
}

Sr. Editor. El día 7 de abril la OMS celebra el Día Mundial de la Salud y la frase que acompaña la celebración es "La salud añade vida a los años".

¿Por qué es importante agregar vida a los años? La mortalidad está asociada con la edad, con bajos niveles de educación en la infancia, pobres condiciones de habitabilidad, inseguridad en la provisión de alimentos $y$, especialmente, con una pobre educación (1). De esta manera, el curso de vida, cumple una función determinante en la producción de enfermedades y en el envejecimiento de las personas.

La salud pública está en pleno cambio de paradigma. Los determinantes sociales de la salud (DSS) reemplazan el enfoque de riesgo de las últimas décadas. En estos determinantes se originan las desigualdades en salud como una brecha en las condiciones en que las personas nacen, se desarrollan y trabajan, crecen y envejecen. Esta perspectiva de los DSS enfatiza que la salud de las personas y sociedades, está influenciada por un conjunto de causas políticas, sociales y económicas que influyen en el curso de vida de las personas.

Perú vive un momento de oportunidad. Una economía en expansión, que en 2012 se calcula llegara a 5,1\% nos habla de un momento favorable para el cambio, en medio de un crecimiento regional que elevó el gasto social de 12,3 a $18,4 \%$ del PBI entre 1990-1991 a 2007-2008 (2). A pesar que estos gastos son "procíclicos" el gasto social en Perú aún esta por debajo del 8\%. La inversión social es fundamental, ya que es el principal recurso de financiamiento para políticas que buscan cortar con la reproducción intergeneracional de la pobreza; un círculo vicioso muy característico de nuestras sociedades latinoamericanas.

\footnotetext{
${ }^{1}$ Organización Panamericana de la Salud (OPS/OMS). Washington DC, EE.UU.

a Médico doctor en Medicina
}

Recibido: 19-04-12 Aprobado: 02-05-12

En este escenario, la educación y la salud forman una pareja indisoluble. Mejor educación se asocia con mejor salud ${ }^{(3)}$. A nivel regional, el gasto en educación pasó de 3,1\% en 1990 a 4,2\% del PBI en 2008; en Perú fue del 1,5 al 2,7\%. En el último examen PISA de 2009, Perú fue uno de los países con los peores resultados de la región, con la mayor proporción de estudiantes con bajo desempeño. Los países que invirtieron más en educación tuvieron mejores resultados; lo notable fue que las brechas fueron mayores al evaluar el ingreso que al evaluar el género o medio urbano frente al rural ${ }^{(4)}$.

En salud no es diferente. En 2008 el gasto en salud fue de $4,5 \%$ del $\mathrm{PBI}$, porcentaje que no varía desde 2000 (la media de la región es de 7,7\%). A pesar de la baja inversión, las últimas décadas mostraron grandes progresos en la salud pública del Perú. La expansión del acceso a los servicios de salud es uno de ellos. A pesar de esto, entre el 20 y $30 \%$ de la población está excluida del sistema de salud ${ }^{(5)}$.

La reducción de las desigualdades y la exclusión son retos que vencer, son parte de la justicia social. Esto debería fortalecer todo ánimo de invertir en las nuevas generaciones ya que ellas seran quienes deban enfrentar las demandas de producción que hagan sostenible el desarrollo de un país, sino que además puedan solventar los desafíos que enfrentará Perú en materia de envejecimiento, sin dudas un motivo para hacer que la inversión se traduzca en un curso de vida que agregue salud a los años; tal como promueve este 2012 la OMS en su Día Mundial de la Salud.

\section{Conflictos de interés}

La opinión vertida en este escrito es personal del autor y no refleja la posición u opinión de la institución a la que pertenece.

\section{REFERENCIAS BIBLIOGRÁFICAS}

1. Ferri CP, Acosta D, Guerra M, Huang Y, Llibre-Rodriguez JJ, Salas A, et al. Socioeconomic factors and all cause and cause-specific mortality among older people in Latin America, India, and China: a population-based cohort study. PLoS Med. 2012;9(2):e1001179.

2. Comisión Económica para América Latina (CEPAL). Panorama Social América Latina 2011 [documento informativo]. Santiago de Chile; CEPAL; 2011.

3. Kaplan GA. Economic crises: some thoughts on why, when and where they (might) matter for health--a tale of three countries. Soc Sci Med. 2012;74(5):643-6.

4. Ganimian AJ, Solano Rocha A. Están al nivel? Como se desempeñaron América Latina y el Caribe en el Programa 
para la Evaluación Internacional de Alumnos del 2009 (PISA)? Washington, DC: Inter American Dialogue; 2011.

5. Alcalde-Rabanal JE, Lazo-González O, Nigenda G. Sistema de salud de Perú. Salud Publica Mex. 2011;53 supl 2:s243-54.

Correspondencia: Zambrini Bernardini Diego

Dirección: 525 Twenty third street, NW, Washington DC, EE.UU Teléfono: 001-202-974-3767

Correo electrónico: bernardd@paho.org

\section{PROBLEMAS EN LA PRESCRIPCIÓN MÉDICA PARA EL ADULTO MAYOR EN PERÚ}

\section{PROBLEMS IN MEDICAL PRESCRIPTIONS FOR THE ELDERLY IN PERU}

\author{
Nelson Rodríguez ${ }^{1, a}$, Iris Puente ${ }^{1, a}$, \\ Antonio Bernabé-Ortiz ${ }^{1, b}$
}

Sr. Editor. Hemos revisado el estudio realizado en Argentina por Regueiro et al. respecto a la prescripción de medicamentos en adultos mayores no institucionalizados; en el cual se encontró que el uso de medicamentos potencialmente inapropiados fue alto. Asimismo, se evidenció la limitada información respecto al tema en dicho país ${ }^{(1)}$.

Al buscar información en las bases nacionales, solo se logró ubicar dos publicaciones relacionadas. La primera, realizada en el Hospital Almenara Essalud entre el 2002-2004, evaluó la calidad de la prescripción de 456 medicamentos en 76 pacientes mayores de 65 años catalogados como frágiles. Los medicamentos que presentaron mayor número de problemas fueron los que actúan a nivel cardiovascular $(63,2 \%)$, gastrointestinal $(28,9 \%)$, sistema nervioso central $(27,6 \%)$ y músculo esquelético (17,1\%). El 90,8\% de los pacientes presentó, por lo menos, un problema calificado como inadecuado en alguno de los diez criterios del Medication Aproppiateness Index (MAl) antes de ser hospitalizados ${ }^{(2)}$.

La segunda, realizada en el mismo hospital el 2009, evaluó problemas asociados con medicamentos en

Escuela de Medicina, Universidad Peruana de Ciencias Aplicadas. Lima, Perú.

a Estudiante de Medicina; ${ }^{\text {b }}$ médico magíster en Salud Pública

Recibido: 21-02-12 Aprobado: 07-03-12 cien adultos mayores hospitalizados. Se observó que el $75 \%$ de la población consumía entre cuatro y cinco medicamentos, de los cuales, los que presentaron mayor prescripción inadecuada fueron los de acción cardiovascular $(35,4 \%)$, sistema nervioso central $(15,4 \%)$, digestivo $(15,4 \%)$, músculo-esquelético $(8,3 \%)$, y productos hematológicos $(7,1 \%)$. Entre los medicamentos con mayor sobreprescripción (uso de medicamentos mayor a lo necesario) y disprescripción (prescripción inadecuada) figuraron el nimodipino, la ranitidina, la aspirina, la digoxina y la warfarina. Las reacciones adversas más frecuentes fueron el sangrado y la hipoglicemia ${ }^{(3)}$.

Según lo expuesto, la prescripción de medicamentos en la población adulta mayor representa un serio problema, más aun con el aumento progresivo de este grupo poblacional que va de la mano con un mayor consumo de medicamentos ${ }^{(4)}$. Resulta importante investigar este tema ya que la información en pacientes institucionalizados es escasa, y en los no institucionalizados es nula. De esta manera, se podrá conocer los factores asociados con la inadecuada prescripción a medicamentos para así evitar las reacciones adversas que deterioran la salud del adulto mayor y que además generan un alto costo en salud pública.

\section{Conflictos de interés}

Los autores declaran no tener conflictos de interés.

\section{REFERENCIAS BIBLIOGRÁFICAS}

1. Regueiro M, Mendy N, Cañás M, Farina HO, Nagel P. Uso de medicamentos en adultos mayores no institucionalizados. Rev Peru Med Exp Salud Publica. 2011;28(4):643-7.

2. Oscanoa T. Calidad de prescripción de medicamentos en pacientes geriátricos. An Fac Med Lima. 2005;66(3):195202

3. Oscanoa TJ. Diagnóstico de problemas relacionados con medicamentos en adultos mayores al momento de ser hospitalizados. Rev Peru Med Exp Salud Publica. 2011; 28(2):256-63

4. Debesa F, Cué M. Los medicamentos y el anciano. Rev Cubana Farm. 1999;33(3):210-4

Correspondencia: Nelson Rodríguez Gueorguiev Dirección: Av. Prolongación Primavera 2390, Lima 12, Perú. Teléfono: (511) 3133333

Correo electrónico: u711653@upc.edu.pe 